\title{
L'Immigration dans les théories contemporaines de la démocratie
}

La part salutaire d'indéterminable

\section{Martin Deleixhe}

\section{OpenEdition}

\section{Journals}

Édition électronique

URL : http://journals.openedition.org/ress/1008

DOI : $10.4000 /$ ress. 1008

ISSN : 1663-4446

Éditeur

Librairie Droz

Édition imprimée

Date de publication : 15 décembre 2011

Pagination : 123-144

ISBN : 978-2-600-01575-2

ISSN : 0048-8046

Référence électronique

Martin Deleixhe, «L'Immigration dans les théories contemporaines de la démocratie », Revue

européenne des sciences sociales [En ligne], 49-2 | 2011, mis en ligne le 01 janvier 2015, consulté le 19 avril 2019. URL : http://journals.openedition.org/ress/1008 ; DOI : 10.4000/ress.1008 


\title{
ATTITUDES ET REPRÉSENTATIONS SOCIALES
}

\section{LES ADOLESCENTS FRANÇAIS ET ITALIENS FACE À LA DIVERSITÉ}

\author{
ALESSANDRO BERGAMASCHI \\ Université Nice Sophia Antipolis - Urmis/Lamhess \\ alessandro.bergamaschi@unice.fr
}

\begin{abstract}
Résumé. La psychologie sociale et la sociologie utilisent beaucoup les concepts de représentation sociale et d'attitudes. Cependant, il existe peu d'études visant à faire ressortir les liens qui subsistent entre ces deux variables. Une enquête comparative faite en France et en Italie a permis d'interroger une population adolescente sur leurs représentations et leurs attitudes envers l'immigration. Le résultat fait ressortir le rapport entre les modes de représentation d'un phénomène et son évaluation. Les représentations sociales sur l'immigration trouvent leurs racines dans les discours nationaux à propos de ce phénomène tandis que l'analyse des attitudes révèle que leur contenu s'insère dans leur espace sémantique. Cette étude suggère d'intégrer la démarche sociologique et psychosociale pour saisir le caractère social des attitudes et comprendre comment les évaluations des phénomènes qui entourent l'individu se développent.
\end{abstract}

Mots-clés : attitude, représentation sociale, méthode comparative, préjugé, immigration

\begin{abstract}
The concepts of social representation and attitudes are widely used by both the social psychology and sociology. However, there are few studies to highlight the links that exist between these two variables. Using a comparative survey conducted in France and Italy an adolescent population was asked about their representations and attitudes towards immigration. The approach followed has permitted to illustrate the links between the ways of representing a phenomenon and evaluate it. Social representations of immigration are rooted in national discourse conducted on this phenomenon, while the analysis of attitudes showed that their content fit into the semantic space of the former. The contribution of the study suggests to integrate psychosocial and sociological approach to capture the social character of attitudes and understand how the evaluations of the social phenomena develop.
\end{abstract}

Keywords: attitude, social representation, comparative method, prejudice, immigration 
Quoique les théories ayant pour objet les «attitudes» et les «représentations sociales » soient nées avec la sociologie, elles se sont ensuite développées dans le cadre de la psychologie sociale, se frayant chacune un chemin autonome. Rares sont les études qui ont essayé d'articuler «attitudes» et «représentations sociales», notamment d'un point de vue empirique, en montrant les mécanismes qui les relient.

L'objectif de cet article est de montrer que les attitudes et les représentations sociales constituent des variables étroitement corrélées, en sorte que c'est au sein de ces dernières que les évaluations que l'individu exprime à l'égard les phénomènes sociaux - ses attitudes - prennent forme. À cet effet, une enquête comparative a été menée en France et en Italie sur la base de deux échantillons de lycéens, français et italiens, invités à exprimer leurs points de vue à l'égard des immigrés et des problèmes posés par l'immigration en général. L’hypothèse poursuivie est que l'explication des phénomènes sociaux - en l'occurrence les réactions des sociétés d'accueil face à l'immigration - dépend en grande partie des représentations sociales qui les structurent cognitivement, lesquelles «autorisent» en effet l'expression d'évaluations spontanées par le biais des attitudes exprimées. Ainsi, le contenu précis des préjugés envers les groupes minoritaires trouve son origine dans un réservoir socio-cognitif donné, procurant aux individus interrogés les arguments sur lesquels ils peuvent appuyer leurs évaluations.

Dans un premier temps, nous nous pencherons sur les aspects théoriques et conceptuels nécessaires à la présentation de ce qui structure notre étude. Puis, nous introduirons l’analyse des données proprement dite en faisant état des caractéristiques méthodologiques de l'enquête. Nous articulerons alors notre étude en deux parties : la première sera consacrée à la présentation des résultats et établira que deux formes distinctes de représentations et d’attitudes envers l'immigration ont cours, suivant l'échantillon analysé et la nationalité des lycéens interrogés; la seconde s’attachera à établir une vision d'ensemble des enjeux socio-politiques suscités actuellement par le phénomène de l'immigration en France et en Italie. 
En conclusion, nous ferons une synthèse des résultats et émettrons quelques suggestions de méthode pour nous émanciper d'un individualisme méthodologique radical qui tend à oublier que les prises de positions de l'acteur social envers l'environnement se greffent sur des processus discursifs qui constituent la trame des rapports sociaux.

\section{ATTITUDES ET REPRÉSENTATIONS SOCIALES La genèse parallèle de deux notions distinctes}

\section{I. LE CONCEPT D'«ATTITUDE»}

Le concept d’attitude donne lieu à des développements scientifiques en des termes très propices à l'analyse sociale. Thomas et Znaniecki qualifient l'attitude de «processus psychologique qui se manifeste en premier lieu envers le monde social et en relation à des valeurs sociales » (1918, p. 26-27). Selon eux, on peut parler d'attitude lorsque quelque chose d'extérieur au monde psychique de l'individu l'interpelle. Au cours des décennies suivantes, et à travers le développement de la statistique sociale, l'approche individualiste s'est imposée (Thurstone, 1928; Bogardus 1931 ; Allport, 1935). L'intérêt des chercheurs s'est alors focalisé sur les dynamiques mentales aboutissant à l'évaluation des stimuli émis par l'environnement. Cette manière d'aborder les attitudes culmine durant les années I960 avec une conception qui, aujourd'hui encore, jouit du plus large consensus. Selon Rosenberg et Hovland, à une attitude correspond l'évaluation d'un objet suivant trois dimensions: affective (les sentiments favorables ou défavorables suscités par le stimulus), cognitive (les perceptions et les croyances), conative (la disposition à l'action, orientée vers le futur ou le passé) (1975, p. 340). Depuis la naissance de ce modèle, enrichi ensuite par les contributions de la social cognition, les études sur les attitudes se sont focalisées sur: a) les liens de cohérence entre ses différentes dimensions internes, b) leur fonction, c) leur capacité à prédire le comportement, d) leur changement. C'est sur la base de ce modèle que les attitudes sont aujourd'hui définies comme l'évaluation psychologique d'un objet selon un certain degré de faveur ou de défaveur (Eagly, Chaiken, 1993). 
En accord avec nos objectifs, il importe de souligner que quelques auteurs affirment que « dans une certaine mesure, [...], l'origine des attitudes est toujours extérieure à l'individu» (Thomas, Alaphilippe, 1993, p. 54; Sherif, 1967; Deconchy, 2003 [1984]). Ce serait alors, selon ces auteurs, l'influence que les groupes primaires et secondaires exercent sur l'individu qui serait à l'origine de la formation des attitudes. Ceci établi, le but de notre contribution dépasse néanmoins le cadre purement interactionniste et individuel. En effet, nous souhaitons souligner que les influences supra-individuelles, contribuant à la construction de la représentation cognitive des phénomènes sociaux, se traduisent dans des réponses en termes d'attitudes communes, abstraction faite des expressions caractéristiques des membres du groupe.

\section{I.2. LE CONCEPT DE «REPRÉSENTATION SOCIALE»}

La notion de représentation connaît un grand essor avec la pensée de Durkheim qui l'appelle «collective» et non pas «sociale», en la définissant comme la «manière selon laquelle cet être spécial qui est la société, pense sa propre expérience» (I968 [I9I2], p. 62I). Cependant, après Durkheim, l’intérêt pour les représentations diminue. Quoique les travaux de Freud (2010 [1905]), Piaget (2005 [1947]) et Levy-Bruhl (1963 [1927]) fassent figure d'exception, ils ne font toutefois pas véritablement progresser les études sur cette question. Il faudra attendre Moscovici pour pouvoir parler d'un véritable regain d'intérêt scientifique envers l'étude des représentations. Ce dernier a pour mérite d’avoir saisi le côté processuel et social des représentations, lesquelles sont le produit de la communication interindividuelle. Leur fonction primaire consiste à «rendre quelque chose d'inhabituel ou l'inconnu lui-même, familier» (Moscovici,1976, p. 38). Le caractère social des représentations relève du fait que les codes de communication sont empruntés au fond culturel commun d'un contexte donné, structuré par des principes, valeurs et idéologies. Il s’agit donc de «modalités de pensée pratique orientées vers la communication, la compréhension et la maitrise de l'environnement social, matériel et idéal »' (Jodelet, 2003, p. 37I). Aussi les représentations se situent-elles à un niveau cognitif élevé, circulant dans la société au moyen du langage et 
des discours. Comme Seca le note, elles constituent des produits culturels «qui naissent et se développent dans les conversations quotidiennes et par rapport à des circonstances culturelles et historiques » (200I, p. I3).

Depuis les travaux d’Abric (1987), l’approche structurelle s’est développée. Selon Abric, les représentations sociales possèdent une structure interne, à savoir un noyau central et un système périphérique. La théorie du noyau central établit que toute représentation prend appui sur un cœur de cognitions à caractère fonctionnel et normatif qui structurent son architecture. Autour du noyau central s'agencent les cognitions dites périphériques, bien plus nombreuses que celles contenues dans le noyau, et qui permettent «l'ancrage de la représentation dans la réalité du moment et présentent une plus grande souplesse que les éléments centraux» (Flament, 1994). En ce qui concerne la socio-dynamique des représentations sociales, il apparaît que lorsque l'environnement entraîne un changement, les cognitions les plus périphériques sont d’abord mises en avant tandis que le noyau n'est sollicité que plus tardivement.

\section{I.3. POUR UNE COMPRÉHENSION SOCIO-PSYCHOLOGIQUE DES PHÉNOMÈNES SOCIAUX}

Les études qui ont essayé d’intégrer dans la même démarche l’analyse des représentations sociales et des attitudes ne sont pas très nombreuses. Elles se déploient soit dans un cadre purement théorique, soit strictement expérimental. Dans le premier cas, nous trouvons le modèle proposé par Moscovici et Vignaux qui assigne à l'attitude une position intermédiaire entre les opinions verbales, réglementant les échanges quotidiens et les représentations. Lattitude possèderait alors la capacité d’adapter ces dernières au réel (Moscovici, Vignaux, 1994). Abric, quant à lui, a mené plusieurs expériences sur la relation entre représentations et comportements, notamment lors de sa recherche sur la «représentation de la tâche» chez un groupe d'étudiants (1994)². De même, pouvonsnous signaler l'analyse de Rateau qui, à partir de la «représentation du groupe idéal», se donne comme objectif de faire ressortir la relation hiérarchique entre idéologie, représentations et attitudes. Tandis que les idéologies préparent

2 Pour une revue des expériences menées, voir Abric (2003). 
le terrain aux représentations, ces dernières font le lit d'évaluations plus spécifiques à la faveur de l'expression d'attitudes (Rateau, 2000). Cependant, il est difficile de ne pas admettre que, dans ces cas, le cadre de référence est toujours celui du stimulus-réponse, la relation entre l'individu et le stimulus présenté dans le cadre d'une expérience étant primordiale. En outre, si nous partons du constat que les représentations sont des «programmes de connaissance sociale » à caractère culturel et discursif, associés à un niveau cognitivement élevé, nous pourrions énoncer certains doutes quant à la présence effective de représentations dans le cadre de telles expériences. Il semblerait que nous soyons plutôt confrontés à de simples réactions individuelles, induites par les stéréotypes attachés à l'objet présenté aux participants lors de ces expériences³.

La démarche que nous avons suivie vise expressément à intégrer les attitudes dans le champ plus vaste des discours publics, produits à un moment donné à propos d’un phénomène précis. Nous montrerons que les représentations sociales provenant de ces discours constituent la toile de fond socio-cognitive sur laquelle les attitudes trouvent leurs racines (sociales) profondes. Nous nous tournerons ensuite vers les représentations sociales relatives aux discours sur l'immigration en France et en Italie. Et nous nous intéresserons alors aux différents effets que de telles attitudes à l'encontre du phénomène de l'immigration induisent, en tâchant de mesurer le niveau de préjugé attaché aux groupes minoritaires. Notre démarche vise donc à ancrer l'analyse des attitudes dans le cadre des rapports sociaux, qui définissent la manière de percevoir la montée de la diversité culturelle et religieuse dans deux contextes donnés. Et de cette manière, nous tâcherons de faire nôtre la réflexion de Jaspars et Fraser. Selon ces auteurs, la valeur de ces études réside dans le fait qu'elles permettent de montrer «to what extent such latent representations are social in the sense that they are shared by individuals belonging to the same social groups » (1984, p. I22). 


\section{MÉTHODOLOGIE DE L’ENQUÊTE}

L'étude s'appuie sur un échantillon de r.org lycéens répartis entre français $(49,1 \%)$ et italiens (50,9\%). L'âge moyen est égal à I7,2 ans. La décision de se focaliser sur une population jeune, encore loin des «rôles adultes» (Galland, 2009, p. 6I-62), est motivée par le fait que l'adolescence représente un moment crucial pour l'étude des attitudes inter-groupes. C'est pendant l'adolescence que les évaluations, singulièrement à l'égard des questions sociales et politiques, se consolident (Ingleart, 1997; Altmeyer, 1998; Reinders, 2003 ; Hooghe, Wilkenfeld, 2008 ; Duriez, Soenens, 2009) car l'individu commence à réfléchir à son futur et à former ses attentes de statut (Galland, 2007, p. 19I-196). Durant ces années, le monde extérieur acquiert progressivement pour lui une signification et il commence à évaluer la relation «Nous-Eux» au prisme de sa position sociale et de ses représentations de la réalité.

Les effectifs ont été sélectionnés dans les lycées professionnels, technologiques et généraux des villes de Nice, pour la France, et de Turin et Gênes, pour l'Italie ${ }^{4}$. Deux variables principales ont servi à l'échantillonnage : a) un statut socio-économique hétérogène, exprimé par la profession des parents, b) une plus ou moins grande densité d'élèves membres de groupes minoritaires inscrits dans les établissements, exprimée par le pourcentage d'élèves étrangers ou nés à l'étranger, puis naturalisés.

La population des deux échantillons est divisée selon le type d'école fréquentée : lycées professionnels (It. 37,I \% - Fr. 39,2 \%), lycées technologiques (It. 29,2\% - Fr. 28,8), lycées généraux (It. 33,3\% - Fr. 32,0\%). Quant au statut socio-économique ses caractéristiques sont les suivantes ${ }^{5}$ : statut bas (It. 42,2\% - 37,I \% Fr.), moyen (It. 37,8\% - Fr. 40,3\%), élevé (It. 20,0\% - Fr. 21,6\%) ${ }^{6}$. Le pourcentage d'étudiants immigrés ou issus de l'immigration oscille entre

4 Le recueil des données a eu lieu aux mois de janvier et février 2009.

5 Le codage de la variable de classe a suivi les lignes indiquées dans la Classification Internationale Type des Professions dans l'Union Européenne (CITP-08 COM).

6 La corrélation entre les trois niveaux scolaires et la classe sociale d'appartenance, donnée par la profession des parents, est forte: Fr 0,54 - It 0,6I (Pearson). 
2, I \% et $25,2 \%$ du total des inscrits pour les établissements sélectionnés en Italie et entre $\mathrm{I}, 4 \%$ et I $2,3 \%$ pour l'échantillon français ${ }^{7}$.

L'analyse des données se concentre sur la population nationale, ou majoritaire, c'est-à-dire qui est composée d’adolescents sans expérience d’immigration et dont la famille est française depuis au moins deux générations. On a exclu de l'analyse des données les adolescents nés à l'étranger ou dont au moins un parent ou un grand-parent a immigré (Baerveld et al., 2004; Vermeij et al., 2009). La démarche adoptée dans cette enquête privilégie donc les évaluations sur les problèmes liés à l'immigration de la part d'une population jeune dont les origines sont exclusivement italiennes ou françaises pour les deux générations précédentes. La notion de population nationale étant au cœur d'un débat actuel méthodologique et épistémologique dont l'issue est incertaine, il convient de préciser que cette recherche ne vise nullement à apporter une contribution d'ordre épistémologique à la question «que signifie être national?» (Tribalat, 1995; Blum, 1997, Le Bras, 1998). Nous avons au contraire pris le parti pragmatique de nous concentrer sur une population dont la mémoire familiale récente n’est pas marquée par des expériences d'immigration. Il est en effet assez contestable de soutenir que les souvenirs et la mémoire familiale d'un adolescent puissent remonter au-delà de la biographie des grands-parents (Dolto, 1989; Ancelin-Schutzenberger, 1997). Par ailleurs, l'enquête entend rendre compte d'un état donné de l'époque contemporaine, en donnant à voir un instantané de la manière dont est perçue l'immigration actuelle par les jeunes citoyens des sociétés d’accueil.

$7 \quad$ Les différences entre les pourcentages sur les élèves étrangers entre les deux échantillons sont dictées par le fait qu'en Italie l'immigration est un phénomène récent, la seconde génération est encore très jeune et la législation nationale en matière de nationalité ne favorise pas l'acquisition ( 10 ans de résidence régulière pour commencer les démarches : loi du 5 février 1992 n91). En France, malgré l'importance historique du phénomène et le nombre élevé d'étrangers qui ont franchi ses frontières, le fait de compter des secondes et des troisièmes «générations d'origine immigrée» et, en particulier, de disposer d'une loi sur la naturalisation orientée principalement au droit de sol (5 ans de résidence pour commencer les démarches: loi du 26 novembre 2003 $n^{\circ}$ 2003-III9), explique que dans les statistiques officielles les étrangers sont paradoxalement moins nombreux par rapport aux pays d'immigration plus récente comme l'Italie.

8 L'échantillon non épuré des cas avec origines étrangères compte en fait un nombre supérieur de cas, égal à 1.506 
La technique de collecte des données se fonde sur un questionnaire semistructuré. Les représentations sociales ont été repérées au moyen d'un test d’association de mots. Afin d'éviter d'influencer les réponses en présentant dès le départ l'objet d'étude comme un problème social, nous avons préféré stimuler la réflexion des jeunes en restant le plus neutre possible. Ils ont donc associé au terme «immigré» les premiers mots qui leur venaient à l'esprit ${ }^{9}$. Ce choix s'est imposé car, comme Sayad le note, «de nombreuses “représentations collectives” » pèsent sur l'immigré qui se trouve au centre des débats sur l'immigration (2006, p. 63) ${ }^{10}$.

L’analyse des attitudes a été, quant à elle, réalisée au moyen de modèles de régression linéaire et logistique. Elle s’articule sur quatre dimensions sémantiques spécifiques d'ordre socio-économique et culturel, typiquement utilisées par les études sur les attitudes inter-groupes. Les deux premières se rapportent à la Théorie des conflits réels qui explore la relation entre le phénomène migratoire et les prérogatives économiques et sociales que le groupe majoritaire se représente en termes exclusifs : l'insertion sur le marché du travail et le droit de vivre dans des espaces non menacés par la délinquance (Sherif 1967; Smith, I98I ; Stephan, 1996). Quant à la troisième et la quatrième dimensions, elles sont ciblées sur des questions d'ordre identitaire. Elles s'inspirent des principes de la Théorie de l'identité sociale, examinant à la fois les réactions face à la montée du pluralisme culturel et religieux (côté socio-culturel) et la propension à octroyer la nationalité aux étrangers (côté naturalisation) (Tajfel, I98I ; Tajfel, Turner, 1986 ; Scheepers et al., 2002). Ces dimensions doivent être lues comme des indicateurs du niveau de «menace $\rangle^{11}$ - de caractère économique, social et culturel - perçue par le groupe majoritaire à l'égard du phénomène de l’immigration.

9 Q. Peux-tu me dire ce qui te vient immédiatement à l'esprit lorsque tu entends le mot «immigré»?

10 Les expressions citées, jusqu'à un maximum de six, ont été analysées au moyen de deux variables principales. La fréquence est donnée par l'importance que chaque catégorie revêt sur le total et est exprimée en pourcentage. Le rang moyen s'exprime en valeurs absolues et a été calculé à travers l'analyse de la variance (anova). Le rang est l'ordre d'apparition des mots, termes ou attributs sur le jeu des associations. Un rang faible signifie que l'énoncé figure sur les premières lignes du test et il fait donc partie du noyau de la représentation (Abric, 1994, 2003).

II De l'anglais «threat». Dans la littérature sur les relations inter-groupes cette expression a été introduite par H. Blumer dans son article de 1958, "Race Prejudice As a Sens of Group Position", Pacific Sociological Review, vol. I, n%. 
L'intérêt de l'étude réside dans la mise en relation des représentations et des évaluations que les deux groupes d'adolescents expriment à propos de ce phénomène quand il est associé à de telles thématiques, compte tenu des caractéristiques qu'il revêt dans les pays respectifs.

\section{RÉSULTATS}

\section{I. LA «REPRÉSENTATION SOCIALE»DE L'IMMIGRÉ} Le réservoir socio-cognitif où les attitudes prennent forme

Tout d'abord, il importe d'établir la toile de fond sur laquelle les attitudes relatives au phénomène de l'immigration prennent forme, en mettant à jour les représentations sociales (RS) de l'immigré qui ont court en France et en Italie. Les deux tableaux suivants illustrent l'agencement des citations (c'est-àdire des expressions citées par les lycéens en réponse au jeu des associations) suivant l'axe noyau central - système périphérique sur lequel se déploie la structure des RS. La lecture et l'interprétation de l'architecture des données mettront l'accent sur les catégories les plus significatives dans la perspective de notre étude.

Graph. I :

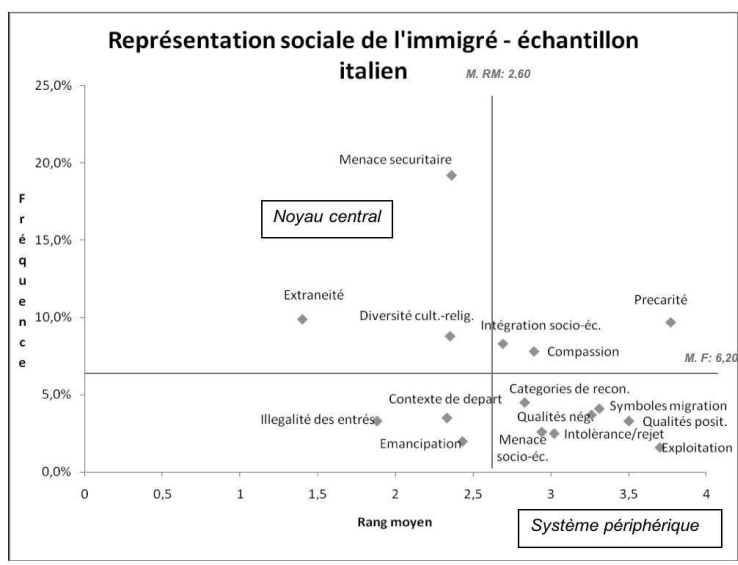

\begin{tabular}{|c|c|c|}
\hline \multicolumn{3}{|c|}{$\begin{array}{l}\text { Echantillon italien } \\
\text { Macro catégories avec Rang moyen et Fréquence }\end{array}$} \\
\hline & Rang moyen & Fréquence \\
\hline Menace sécuritaire & 2,36 & $19,2 \%$ \\
\hline Extranéité & 1,4 & $9,9 \%$ \\
\hline Diversité cult. et relig. & 2,35 & $8,8 \%$ \\
\hline Précarité & 3,77 & $9,7 \%$ \\
\hline Rôles socio-éco. & 2,69 & $8,3 \%$ \\
\hline Compassion & 2,89 & $7,8 \%$ \\
\hline Contexte de départ & 2,33 & $3,5 \%$ \\
\hline Illégalité des entrées & 1,88 & $3,3 \%$ \\
\hline $\begin{array}{l}\text { Catégories de } \\
\text { reconnaissance }\end{array}$ & 2,83 & $4,5 \%$ \\
\hline Symboles de la migrations & 3,31 & $4,1 \%$ \\
\hline Qualités négatives & 3,26 & $3,7 \%$ \\
\hline Qualités positives & 3,5 & $3,3 \%$ \\
\hline Intolérance/rejet & 2,94 & $2,6 \%$ \\
\hline Menace socio-éco. & 3,02 & $2,5 \%$ \\
\hline Émancipation & 2,43 & $2,0 \%$ \\
\hline Exploitation & 3,7 & $1,6 \%$ \\
\hline
\end{tabular}

M. RM : moyenne rang moyen

M. $F$ : moyenne fréquence

Les scores utilisés pour définir la fréquence de citation haute ou basse et le rang moyen d'apparition faible ou fort dérivent de la moyenne calculée pour chacune catégorie sur l'ensemble de la population. 
Graph. 2 :

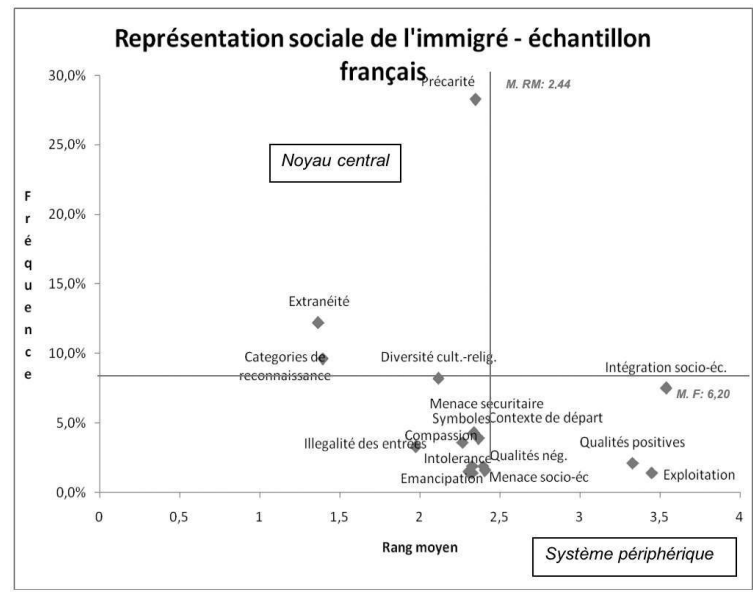

\begin{tabular}{|c|c|c|}
\hline \multicolumn{3}{|l|}{ Echantillon français } \\
\hline & Rang moyen & Fréquence \\
\hline Précarité & 2,35 & $28,2 \%$ \\
\hline Extranéité & 1,37 & $12,2 \%$ \\
\hline $\begin{array}{l}\text { Catégories de } \\
\text { reconnaissance }\end{array}$ & 1,4 & $9,6 \%$ \\
\hline Diversité cult. et relig. & 2,12 & $8,2 \%$ \\
\hline Rôles socio-éco. & 3,54 & $7,5 \%$ \\
\hline Menace sécuritaire & 2,34 & $4,3 \%$ \\
\hline Contexte de départ & 2,37 & $3,9 \%$ \\
\hline Symboles de la migration & 2,27 & $3,6 \%$ \\
\hline Illégalité des entrées & 1,98 & $3,3 \%$ \\
\hline Émancipation & 2,33 & $1,9 \%$ \\
\hline Menace socio-éco. & 2,4 & $1,9 \%$ \\
\hline Qualités négatives & 2,41 & $1,6 \%$ \\
\hline Intolérance/rejet & 2,31 & $1,5 \%$ \\
\hline Compassion & 2,33 & $1,4 \%$ \\
\hline Qualités positives & 3,33 & $2,1 \%$ \\
\hline Exploitation & 3,45 & $1,40 \%$ \\
\hline
\end{tabular}

M. RM : moyenne Rang moyen

$M . F$ : moyenne fréquence

Les scores utilisés pour définir la fréquence de citation haute ou basse et le rang moyen d'apparition faible ou fort dérivent de la moyenne calculée pour chacune catégorie sur l'ensemble de la population

En ce qui concerne le groupe italien, la RS de l'immigré est axée sur une idée de «menace sécuritaire», ne nécessitant pas de longs commentaires (fréquence I 9,2 \% - rang moyen 2,36). Pour les adolescents français, au contraire, cette caractéristique présente peu d'importance, et c'est surtout l'idée de «précarité » qui domine dans leur RS de l'immigré (avec des valeurs bien supérieures à la donnée italienne : 28,2 \% du total contre 9,7\%) avant tout une personne en proie à des difficultés en matière de logement, de carrière professionnelle, de qualité de vie dans certains quartiers, etc., nuisant à la qualité de son intégration sociale. Une autre différence intéressante réside dans la disparité des cognitions situées dans la zone la plus périphérique des RS, où les citations sont caractérisées par la logique de basse fréquence et de rang moyen élevé. On peut qualifier, avec Seca, ce type de réponses de «référents linguistiques lors des modulations conversationnelles », tels que la RS de l'immigré peut se propager dans le discours (op.cit., p. II3). Le faible nombre d’attributs énoncés par les jeunes Français semble suggérer que, pour eux, l’immigré - et les questions qu’il 
suscite - ne représentent pas un sujet de débat «à la une » dans le discours public. Cette constatation contraste avec le fait que les cognitions renvoyant à l'idée de «diversité $»^{13}$ occupent un espace central dans les noyaux des deux représentations. En outre, il est remarquable que dans le noyau de la RS du groupe français émerge également une tendance à catégoriser l'immigré (suivant des «catégories de reconnaissance $\gg^{{ }^{4}}$ ), alors que ces cognitions glissent dans le système périphérique de la RS élaborée par le groupe italien; dans le paragraphe 4.2. nous tâcherons d'approfondir l'examen de cette apparente contradiction. Pour le groupe italien, au contraire, les éléments constitutifs de la RS de l'immigré prennent forme dans son aire plus externe et discursive.

Enfin, un autre ensemble de cognitions mérite d'être abordé. Il s'agit de la catégorie dite de «compassion», perceptible à travers un ensemble de citations relatives à une vision compassionnelle de l'immigré. Ces cognitions sont situées dans la partie externe du noyau central de la RS du groupe italien. Quant au groupe français, cette vision revêt une importance manifestement moindre, puisque c'est une cognition qui se situe dans la zone la plus périphérique de la RS. Ce concept ne se réfère pas à la simple disposition d'ouverture face à la diversité, mais concerne les cognitions qui identifient les migrants comme des individus malheureux, à l'égard desquels il convient de faire montre de patience et d'indulgence, et qu'il faut secourir à tout prix : car en réalité, «Eux » ont besoin que «Nous» les aidions. Par conséquent, il serait possible d'interpréter un tel écart entre les résultats comme une manière implicitement différente de percevoir l'immigré de la part des deux groupes de lycéens interrogés.

En conclusion, il est à noter que les deux structures cognitives que nous venons de présenter ont été décrites avec comme seule variable indépendante la nationalité des lycéens interrogés. Aucune autre variable dépendante de caractère contextuel (ex. le statut économico-culturel de la famille) ou individuel (ex. le

13 Cette catégorie inclut les associations qui évoquent les différences d'us et coutumes, de traditions et de religions.

14 II s'agit des catégorisations habituellement employées dans le discours public pour identifier les immigrés selon leurs origines nationales, «ethniques» ou les autres attributs phénotypiques (ex. Noir, Arabe, Asiatique, Marocain, Roumain, etc.). 
nombre d'amis membres de groupes minoritaires) ne produit une variation statistiquement significative dans les fréquences et les rangs d’apparition des cognitions qui composent les deux RS. Aussi, sommes-nous face à deux structures, attachées à un niveau socio-cognitif élevé, qui sont probablement le reflet de la dimension nationale uniquement. Confronté à ce résultat, nous pouvons commencer à nous demander si cette disparité ne découle pas de deux manières différentes de percevoir le statut de la diversité dans l'espace public de ces deux pays en question.

Aussi, après avoir présenté les points essentiels de la RS de l'immigré, élaborée selon les deux échantillons, pouvons-nous désormais poursuivre l'analyse en illustrant ce qui en résulte au moyen des indicateurs utilisés pour l'étude des attitudes.

\subsection{LES ATTITUDES ENVERS LE PHÉNOMÈNE DE L'IMMIGRATION}

La première dimension concernant l'étude des attitudes envers l'immigration reste économique. Dans ce cas, le groupe majoritaire exprime des attitudes défavorables à l'égard des groupes minoritaires parce qu'il redoute que ces derniers puissent menacer ses droits et ses privilèges de caractère économique et social (et, en priorité, son accès au marché du travail). Le tableau suivant présente le résultat relatif à l'indicateur utilisé pour étudier cette dimension, à savoir la question suivante : «À ton avis, l’immigration et ses effets sur la société peuvent-ils influencer la réalisation de tes projets d'avenir? $\gg^{15}$.

Tableau I :

\begin{tabular}{|c|c|c|c|c|c|c|}
\hline $\begin{array}{c}\mathrm{P}^{* * * *} \leq .001 \\
\mathrm{P}^{* *} \leq .010 \\
\mathrm{P}^{*} \leq .050\end{array}$ & $\begin{array}{c}\text { COEFFICIENTS NON } \\
\text { STANDARDISÉS }\end{array}$ & $\mathrm{B}$ & $\begin{array}{r}\text { ERREUR } \\
\text { STANDARD }\end{array}$ & $\begin{array}{c}\text { COEFFICIENTS } \\
\text { STANDARDISÉS }\end{array}$ & & \\
\cline { 1 - 7 } MODĖLE & & & & Beta & T & SIG. \\
\hline \multirow{2}{*}{$\mathrm{I}$} & (Constant) & 6,004 &, 109 & & 54,951 &, 000 \\
\cline { 2 - 7 } & Pays*** & $-2,086$ &, 205 &,- 345 & $-10,167$ &, 000 \\
\hline
\end{tabular}

$N: 851$ (nombre des effectifs totaux inclus dans l'équation de régression linéaire) $R^{2} 0,268$

I5 La variable dépendante est numérique, comptant 10 modalités de réponses comprises entre I aucune influence - 10 c'est un obstacle. 
Ce tableau indique que les lycéens italiens sont plus enclins à accuser l'immigration d'entrave à la réalisation de projets d’avenir professionnels ${ }^{16}$. Cette lecture émerge du fait que l'indicateur choisi, oriente la réflexion vers un niveau typiquement égotropique ${ }^{17}$, de telle sorte que les interrogés ont dû mettre en relation ce phénomène avec les perceptions et les attentes relatives à leur avenir personnel.

Lorsque nous passons de la «dimension économique » aux deux indicateurs de la «dimension sécuritaire», le cadre suivant se profile. S’agissant du premier indicateur, qui vise à relever ce que l'on a appelé la tendance à surestimer le nombre des immigrés, le tableau suivant montre que le groupe italien est le plus porté à percevoir son pays comme peuplé par un nombre d'étrangers bien supérieur à la réalité (Q. «Selon toi, combien y a-t-il d'étrangers en France/Italie?»)18.

Tableau 2:

\begin{tabular}{|c|c|c|c|c|c|c|c|}
\hline \multicolumn{2}{|c|}{$\begin{array}{l}\mathrm{p}^{* * * *} \leq .001 \\
\mathrm{p}^{* *} \leq .010 \\
\mathrm{p}^{*} \leq .050\end{array}$} & \multirow{2}{*}{$\frac{\text { B }}{-1,942}$} & \multirow{2}{*}{$\begin{array}{l}\text { E.S. } \\
, 181\end{array}$} & \multirow{2}{*}{$\frac{\text { Wald }}{114,532}$} & \multirow{2}{*}{$\frac{D f}{1}$} & \multirow{2}{*}{$\begin{array}{l}\text { Sig. } \\
, 000\end{array}$} & \multirow{2}{*}{$\frac{\text { RC }}{143}$} \\
\hline Step $I^{a}$ & Pays*** & & & & & & \\
\hline & Constant & ,935 & ,095 & 97,413 & I &, 000 & 2,548 \\
\hline
\end{tabular}

$N: 863$ (nombre des effectifs totaux inclus dans l'équation de régression logistique)

R2 Nagelkerke 0.322

Pourcentage global $72.2 \%$

$R C$ : rapport de cotes (odds ratio)

16 En ce qui concerne le codage de la variable indépendante, c'est-à-dire le pays de résidence, nous avons codé avec I l'échantillon de lycéens français et avec 0 l'échantillon italien.

17 Pour une discussion sur la distinction des niveaux égotropiques et sociotropiques dans l'étude quantitative des attitudes voir Mc Laren (2003); voir aussi la distinction entre «abstract prejudice» et «personal prejudice» proposée par Weldon (2006, p. 345) et reprise par Dejaghere, Hooghe, Claes (2011, p. 4).

I8 L'item compte 6 modalités de réponse: a. moins de 2 millions, b. entre 2 et 4 millions, c. entre 4 et 6 millions, d. entre 6 et 8 millions, e. plus de 10 millions. Le but étant de faire ressortir le groupe ayant le plus de probabilité à surestimer la dimension du phénomène, pour l'analyse des données cette variable a été ensuite recodée en 2 modalités principales, l'une concernant la sous-estimation et l'estimation correcte (a. b.: codée avec 0) l'autre concernant la surestimation (c. d. e. f.: codée avec I). 
Il en résulte que l'estimation formulée par l'échantillon italien peut être 7 fois supérieure à celle émise par l'échantillon français ${ }^{19}$. Un tel écart s'explique par le recours à un cadre de référence fort différent de celui du groupe français, dont les perceptions se placent sur un registre correspondant davantage à la situation réelle ${ }^{20}$. En outre, il convient de souligner que, dans ce cas, la seule variable indépendante réussit à expliquer un tiers de la variance du modèle.

Avec le deuxième indicateur portant sur la «dimension sécuritaire», nous pénétrons au cœur du binôme épineux immigration-criminalité (Q. «En général, penses-tu que l'immigration a contribué à l'augmentation de la délinquance en France/Italie?») ${ }^{21}$. Le tableau présenté ci-dessous permet de constater que l'échantillon italien est le plus enclin à accuser l'immigration d'avoir augmenté la criminalité dans son pays.

Tableau 3:

\begin{tabular}{|c|c|c|c|c|c|c|}
\hline $\begin{array}{c}\mathrm{P}^{* * * *} \leq .001 \\
\mathrm{P}^{* *} \leq .010 \\
\mathrm{P}^{*} \leq .050\end{array}$ & $\begin{array}{c}\text { COEFFICIENTS NON } \\
\text { STANDARDISÉS }\end{array}$ & $\mathrm{B}$ & $\begin{array}{r}\text { ERREUR } \\
\text { STANDARD }\end{array}$ & $\begin{array}{c}\text { COEFFICIENTS } \\
\text { STANDARDISÉS }\end{array}$ & & \\
\hline MODĖLE & & & & Beta & T & SIG. \\
\hline \multirow{2}{*}{$\mathrm{I}$} & (Constant) & 1,762 &, 037 & & 47,779 &, 000 \\
\cline { 2 - 8 } & Pays*** &,- 237 &, 068 &, 124 & 2,015 &, 018 \\
\hline
\end{tabular}

$N: 873$ (nombre des effectifs totaux inclus dans l'équation de régression linéaire) $R^{2} 0,117$

Cependant, les coefficients de significativité et de variance expliqués sont moins robustes par rapport aux modèles précédents ( $\mathrm{p}^{*} ; \mathrm{R}^{2}$ o.II7), signe que la question «immigration-criminalité » est un enjeu à la une, tant dans le contexte italien que français. Cette observation faite, si nous considérons la «dimension sécuritaire» dans sa globalité, nous pouvons constater que les

19 La formule appliquée pour calculer la probabilité estimée est la suivante: I/Exp(B): I/0.143 = 6.9937; d'après Demaris (1992, p. 46-48).

20 Pour ce qui est de la situation italienne, les dernières estimations relatives à la période où le recueil empirique a eu lieu comptent un nombre d'étrangers égal à 3.987.112 personnes (Caritas/Migrantes, 2008, p. 95). Pour la réalité de l'Hexagone, 3.561.200 de non-Français ont été enregistrés à la même époque, (Institut National des Études Statistiques et Économiques, 2007: http://www.insee.fr/fr/themes/tableau.asp?reg_id=0\&ref_id=NATTEF02 I3I).

21 L'item compte 4 modalités de réponse: a. oui beaucoup, b. oui assez, c. un peu, d. non pas du tout. 
lycéens de la Péninsule se démarquent par l'expression d’attitudes plus négatives à l'égard de l'immigration.

$\mathrm{La}$ « dimension socio-culturelle » vient clore notre présentation des résultats, à la faveur de deux indicateurs supplémentaires. Le premier met l'accent sur la présence du pluralisme culturel et religieux (Q. a. «Les immigrés enrichissent la culture française/italienne »; b. «Dans les Mosquées il y a trop souvent un endoctrinement politique »; c. «Les écoles devraient consacrer plus de temps à l'enseignement de l'histoire et de la culture des immigrés»; d. «Les femmes musulmanes devraient être autorisées à porter le foulard au travail ou à l'école»; e. «Les comportements des immigrés ne sont pas vraiment compatibles avec nos traditions ») $)^{22}$. Le second vise à évaluer la propension des lycéens à octroyer la citoyenneté aux étrangers (Q. «Et en définitive, est-il juste selon toi qưun étranger qui vit en Italie/France puisse devenir italien/ français?»). Le résultat du premier indicateur est illustré dans le tableau qui suit.

Tableau 4:

\begin{tabular}{|c|c|c|c|c|c|c|}
\hline $\begin{array}{c}\mathrm{p}^{* * *}{ }^{*} \leq .001 \\
\mathrm{p}^{* *} \leq .010 \\
\mathrm{p}^{*} \leq .050\end{array}$ & $\begin{array}{l}\text { COEFFICIENTS NON } \\
\text { STANDARDISÉS }\end{array}$ & B & $\begin{array}{c}\text { ERREUR } \\
\text { STANDARD }\end{array}$ & $\begin{array}{l}\text { COEFFICIENTS } \\
\text { STANDARDISÉS }\end{array}$ & & \\
\hline MODÈLE & & & & Beta & $\mathrm{T}$ & SIG. \\
\hline \multirow[t]{2}{*}{ I } & (Constant) & 7,264 &, 113 & & 64,202 & ,000 \\
\hline & Pays*** & ,377 &, 121 & , 147 & 1,306 &, 003 \\
\hline
\end{tabular}

$N: 841$ (nombre des effectifs totaux inclus dans l'équation de régression linéaire) $R^{2} 0,217$

S’agissant des attitudes envers les différences culturelles et religieuses, le groupe de lycéens français apparaît comme davantage disposé à accuser les groupes minoritaires de nuire à la «pureté» de l'identité nationale (tab. 4), à travers leurs us et coutumes. Même constat quant à la disposition à octroyer la citoyenneté (tab. $5)^{23}$. Par ailleurs, les jeunes français, exprimant la crainte que la diversité ethno-

22 Les items a. et d. ont été empruntés au questionnaire utilisé par l'International Social Survey Programm 2003 (2003, p. 27); cette variable a été transformée dans un indice cumulatif, comptant sur un alpha de Cronbach solide égal à $0.7 \mathrm{l}$.

23 Dans ce cas la variable dépendante, c'est à dire les réponses positives ou négatives à l'octroi de la citoyenneté aux étrangers, a été codée avec 0 pour les réponses négatives et I pour les réponses positives. 
culturelle puisse «polluer» les traits fondateurs de l'identité culturelle de leur pays, deviennent par conséquent plus réticents à l'octroi de la citoyenneté.

Tableau 5 :

\begin{tabular}{|c|c|c|c|c|c|c|c|}
\hline \multicolumn{2}{|c|}{$\begin{array}{l}\mathrm{P}^{* * * *} \leq .001 \\
\begin{array}{r}\mathrm{P}^{* *} \leq .010 \\
\mathrm{P}^{*} \leq .050\end{array}\end{array}$} & B & E.S. & Wald & Df & Sig. & RC \\
\hline \multirow{2}{*}{ Step I I } & Pays*** &,- 386 &, 180 & 4,564 & I &, 008 & 1,470 \\
\cline { 2 - 8 } & Constant &, 682 &, 090 & 57,204 & । &, 000 & 1,978 \\
\hline
\end{tabular}

$N: 861$ (nombre des effectifs totaux inclus dans l'équation de régression logistique)

R2 Nagelkerke 0.344

Pourcentage global $72.2 \%$

$R C$ : rapport de cotes (odds ratio)

\section{PANORAMA DES ENJEUX SOCIO-POLITIQUES AUTOUR DE LA QUESTION DE L'IMMIGRATION EN FRANCE ET EN ITALIE}

La France et l'Italie sont deux pays européens hautement concernés par le phénomène de l'immigration, mais selon des caractéristiques fortes qui sont différentes. Un état des lieux sur la «question migratoire» de ces deux pays nous permettra de d’interpréter plus finement nos résultats.

\section{I. L'IMMIGRATION EN ITALIE}

Des préjugés envers un phénomène peu connu

L'Italie n’a été confrontée à l'immigration internationale que récemment, suite à un passé émigrant très important (Fondazione Migrantes, 2006, p. I6). Après seulement trente ans d'immigration, à la fin de l'année 2009, la population étrangère frôle les 5 millions (Caritas/Migrantes, 20ı, p. I07). Il s'agit dans l'ensemble d'un changement soudain et difficile à assimiler de la part de la population nationale (Bolaffi, 200I, p. 3-5).

À partir de la fin des années 1990, l'Italie semble vivre, en matière d'immigration, une période d'«urgence». L'image de l'immigré fusionne avec celle du criminel et l'immigration devient la «métaphore sociale de la déviance» (Dal Lago, I999, p. 30-3I). C’est le parti de la Ligue du Nord qui pilote la poli- 
tique italienne en matière d'immigration, fort notamment de son monopole sur le Ministère de l'Intérieur (Dal Lago 2004, p.7I). Cette situation perdure avec des hauts et des bas au cours des années suivantes, à tel point que certains observateurs externes comme Calavita n'hésitent pas à affirmer qu'en Italie l'immigration est traitée comme l'un des principaux «maux sociaux» qui affligent le pays (Calavita, 2005). Le discours anti-immigration est, en outre, largement répandu grâce à l'action amplificatrice des médias. Morcellini a bien mis en évidence la tendance des médias italiens à «se contenter d’une image statique et apparemment immuable» des immigrés, marquée par le «langage du crime, les expressions de la douleur, les peurs de l'invasion» (2009, p. 27). La spirale de criminalisation de l'immigré a débouché en 2009 sur l'approbation du Pacchetto Sicurezza par le gouvernement Berlusconi, qui enferme complètement la gestion de l'immigration dans le cadre de la maîtrise de la sécurité et de l'ordre public (Loi $\mathrm{n}^{\circ} 94$ du I5 juillet 2009). L’une des dispositions du texte paraît symptomatique de la politique sécuritaire qui sévit dans la Péninsule : l’institution de rondes urbaines actives au niveau de la ville et dépendantes du maire, assurées bénévolement par des associations ou des ex-militaires à la retraite dans le but de renforcer les actions de surveillance en milieu urbain (art. 3 C. 4I-2).

Cependant, ces positions hostiles à l'immigration doivent se confronter aux opinions contraires d'un ensemble d'acteurs de la société civile, se distinguant par une mission et une identité particulières. En Italie, les pouvoirs publics mettent en œuvre les politiques d'intégration en les déléguant massivement aux acteurs privés, parmi lesquels les organisations catholiques jouent un rôle de premier plan (Caponio, 2006, p. 78-85). Dans le débat sur l'immigration, ces «institutions solidaristes » ont été capables de réunir un véritable «front pro-immigrés $»^{24}$ qui, participant pleinement aux politiques d'insertion, vise à protéger la dignité et l'intégrité de la personne, abstraction faite de son origine et, notamment, de la validité de ses papiers (Zincone, 2005, p. 395). On peut dire qu’au niveau rhétorique, l'Église opère une véritable superposition de l'image du «migrant» et de celle de l'individu en détresse, qui demande à être secouru à tout prix. Bien 
que son rôle dans le débat sur l'immigration fasse aussi l'objet d'évaluations plus critiques, il est difficile de ne pas admettre que celle-ci représente un véritable amortisseur social entre la société civile et la classe politique au sujet des questions soulevées par ce phénomène (Ambrosini, 2005, p. 223-229).

Cet aperçu du discours italien sur la «question migratoire » contribue à l'élaboration de la RS de l'immigré qui a cours au sein de l'échantillon des lycéens italiens. La «menace sécuritaire», le pilier de ce réservoir sémanticocognitif, est bien enracinée dans un discours public qui fait du phénomène de l'immigration la cause principale des problèmes du pays. De même avons-nous observé que les réponses en termes d'attitude des lycéens italiens sont visiblement polarisées par une menace à caractère social et économique. C'est donc une vision «fantasmatique » de l'immigration qui s'impose, face à un phénomène dont il faut bien se protéger en délimitant ce qui est à «Nous».

Nous pourrions objecter que ce cadre conflictuel propre à l'Italie est contraire au résultat selon lequel le groupe italien serait plus enclin à répondre par l'affirmative à l'octroi de la citoyenneté. Cette contradiction ne survient toutefois qu'en première lecture. En effet, comme nous l'avons vu plus haut, le noyau de la RS de l'échantillon italien abrite un ensemble de cognitions de type compassionnel, où le migrant est présenté comme une personne en quête d’aide et qu'il faut secourir. Ces cognitions se rapprochent, nous semble-t-il, de la position de l'Église italienne et de sa doctrine sur la mobilité humaine. Malgré les menaces pesant sur les registres socio-économiques, l'accueil des plus vulnérables n’est pas pour autant mis à l'écart - toutefois, sans que cela soit interprété comme une volonté d'intégration. En soutien à l'hypothèse que les «attitudes des jeunes» sont redevables, dans une certaine mesure, du «discours de l'Église », on remarquera que les lycéens de la Péninsule, par rapport à leurs camarades français, sont plus intégrés dans la sphère du sacré, tant du point de vue du sentiment de religiosité personnelle que de la participation aux rites ${ }^{25}$. Si les jeunes italiens sont plus

25 Aussi bien pour la participation aux rites religieux que pour le sentiment de religiosité personnel, l'échantillon italien montre des valeurs nettement plus élevées que celles des adolescents français. Dans les deux cas, la différence est statistiquement significative et avec des écarts solides : a) participation aux rites, $p \leq 0.000$ - phi 0.19 , b) sentiment de religiosité 
religieux, il est probable que les messages de l'Église à l'égard des phénomènes sociaux comme l'immigration soient également mieux intériorisés par rapport au groupe français. Ce dernier est en effet marqué par une intégration religieuse plus faible et situé dans un contexte où l'emprise du discours catholique sur la mobilité humaine est plus modeste (Schor, 1996, p. 259).

\subsection{L'IMMIGRATION EN FRANCE} Des préjugés enracinés envers un phénomène connu

La France est au contraire un pays dont le passé d'immigration est important. Aujourd’hui, comme Bréchon et Tchernia le remarquent, «le grand débat qu’a connu la France depuis le début des années 1980 sur la place des immigrés et des étrangers n’est pas dépassé mais il est beaucoup moins vif quautrefois » (2009, p. 319). Les généralités abusives de l'époque ont laissé place à une manière plus indirecte d'aborder ce sujet, riche en sous-entendus et portant sur des questions plus complexes et aiguës. Ce sont les principes d'égalité et de laïcité qui, interprétés d'une manière universaliste, constituent le socle de toute politique sur l'intégration et de tout débat sur l'immigration. En vertu des principes républicains, les particularismes culturels et religieux des groupes minoritaires ne sont pas pris en compte dans l'espace public (Schnapper, 2008, p. I33).

Le respect de tels préceptes républicains, à caractère national, conduit à des «politiques identitaires» aux conséquences évidentes. Pensons, par exemple, à l'institution du Ministère de l'Immigration et de l'Identité Nationale ${ }^{26}$, pendant la présidence Sarkozy, à l'origine du «Grand Débat sur l'Identité Nationale» qui a pris corps en $2009^{27}$. Une telle accentuation de la dimension identitaire a pour effet de stigmatiser tout groupe qui, en raison de ses spécificités culturelles et de la manière de se comporter dans l'espace public, s'écarte des principes républicains. Emblématiques, à cet égard, sont les enjeux liés à l’islam, où l'accent est mis principalement sur la question du voile ou des lieux de culte.

personnelle, $\mathrm{p}<0.000-\mathrm{F} 43.6$.

26 Puis «Développement solidaire ». Ministère supprimé lors du remaniement gouvernemental en novembre 2010.

27 Les actualités du Grand Débat sur l'Identité Nationale peuvent être retrouvées sur le portail du Gouvernement: http://www.debatidentitenationale.fr/ (consulté le 2 juin 20I0). 
Selon Lapeyronnie, l'islam suscite fréquemment des désapprobations marquées, notamment parce qu'il est associé au «monde des Arabes ou des musulmans, au monde non moderne ou antimoderne de l'Autre» (2008, p. 527). Force est de constater que ces questions représentent des enjeux hautement politisés et médiatisés (Le Goaziou, 2006, p. 43-45). Par ailleurs, la République est confrontée aujourd'hui à des formes d'auto-organisation des collectivités musulmanes qui prônent la cause d'une meilleure intégration et plaident pour une plus grande reconnaissance. C'est le cas du Mouvement des Indigènes de la République qui vise à défier «les valeurs “trompeuses” dont la France se glorifie» tout en dénonçant le caractère fallacieux de son égalitarisme universel (Streiff-Fénart, 2009, p. 234).

Ce dernier point constitue l'épine la plus douloureuse dans le flanc du modèle d'intégration français. L'étude d'Affif, par exemple, tend à montrer que, sur une période de trente ans de traitement médiatique de l'immigration en France, «les représentations de l'immigré souffrantes et doloristes dominent largement» (2004, p. 272). À partir des années I990, la lutte contre les discriminations, «auparavant apanage de la gauche et des organisations antiracistes », est devenue un thème largement consensuel (Noiriel, 2009, p. I75). Les doutes sur l'efficacité du modèle républicain ont atteint leur sommet en 2005 avec les trois semaines de révoltes dans les banlieues des grandes villes françaises; émeutes qui d’ailleurs n'étaient pas les premières (Morice, 2005). Celles-ci ont présenté aux yeux du grand public la plus grave crise d'intégration sociale frappant les 4,7 millions de personnes qui résident dans les 75I «zones urbaines sensibles » de France (Mucchielli, 2006, p. 24). L’importance accordée par le monde des médias et de la politique à ces événements a sans doute contribué à renforcer l’image de l'«immigré non intégré », dont les comportements sont contraires à la façon de vivre dans l'Hexagone, dans l'opinion publique française. Et de fait, depuis quelques années, la question des banlieues semble s’être imposée «durablement dans le paysage social français » (Lapeyronnie, op.cit., p. Io).

À l'instar de l'analyse précédente de la situation italienne, nous pouvons à présent mettre en relation l'état des lieux de l'immigration en France avec les représentations et les attitudes envers ce phénomène. Après un siècle et demi de flux dirigés vers le sol de la République, pour ne parler que de l'époque 
contemporaine, la classe politique prend conscience de la nécessité de s'interroger sur les problèmes posés par la discrimination et la non-intégration de la population immigrée, ce qui semble avoir des incidences directes sur la RS formulée par les lycéens français. En effet, c'est d’abord sur la catégorie de la «précarité » que se structure l'image de l'immigré.

Par ailleurs, nous pouvons constater que le noyau de la RS est investi de catégories normalement utilisées pour distinguer et classer la diversité (noirs, arabes, maghrébin, etc.), bien que celles-ci n'aient guère d'importance dans l'aire discursive la plus externe de la représentation (le système périphérique). Sur ce point, il se peut que la RS de l'immigré ressente l'emprise du modèle républicain d'intégration, fortement ancré dans l'idée d'égalité et de non-reconnaissance des particularismes. En effet, un tel accent mis sur la dimension identitaire a pour effet pervers d'accroître la visibilité des diversités, en stigmatisant tout groupe qui, en raison de ses spécificités culturelles et de la manière de se comporter dans l'espace public, s'écarte des principes républicains, et en l'accusant ainsi de dérive communautariste.

En cohérence avec cet arrière-plan socio-cognitif, le groupe français ne dirige pas ses attitudes négatives sur fond d’enjeux matériels. Les principaux questionnements s'orientent plutôt vers une réflexion plus complexe quant à l'état de l'intégration de la population immigrée et l'enjeu du pluralisme culturel et religieux dans la société française. Cela notamment au vu des demandes de reconnaissances avancées par certains groupes qui tentent de passer de la condition d'outsiders à celle d'insiders. L'immigration déclenche alors la perception d'une menace à caractère identitaire aux yeux des adolescents de l'Hexagone, au point qu'une partie d'entre eux répugne à octroyer à l'immigré le droit à la naturalisation, marquant par là leur volonté de ne pas reconnaître la diversité. Ce préjugé est probablement hérité d’une identité nationale solidement ancrée dans l'histoire contemporaine et structurant la vie publique, ce qui n'est pas le cas dans la situation italienne. L'idée de précarité qui ressort de la RS de l’immigré émerge alors d'un stéréotype plus englobant, où les difficultés sociales témoignant d'une intégration précaire vont de pair avec l'idée d’une incompatibilité culturelle. 


\section{CONCLUSION}

La démarche visant à promouvoir l'analyse des attitudes conjointement à une mise à jour des représentations sociales nous a permis de nous émanciper des seules variables situationnelles et interactionnelles. Ajoutons que dans le cadre de cette étude, la mesure des attitudes tend à montrer que les effets des variables telles que le statut socio-économico-culturel et d’autres propriétés à caractère plus individuel, comme les relations amicales avec les membres des groupes minoritaires, produisent des effets conformes aux attentes : à mesure que les ressources socio-économico-culturelles baissent et que le réseau social de l'acteur est ethniquement plus homogène, le niveau de préjugé enregistre une hausse dans chacune des diverses dimensions considérées. Ceci étant, nous pouvons remarquer que le groupe italien se démarque toujours par un plus fort préjugé à caractère socio-économique alors que le groupe français est davantage concerné par un préjugé de type identitaire. La «stabilité» de cette polarisation s'explique par la présence de deux «théories naïves » différentes, trouvant leur origine dans les discours sur l'immigration développés en France et en Italie. Celles-ci représentent le réservoir socio-cognitif permettant aux jeunes gens interrogés de prendre position sur ce phénomène. Et en effet, comme Rateau le souligne, «la raison même d'une attitude est à chercher en-dehors de celle-ci, à un niveau supérieur dont elle dérive» (Rateau, 2000, p. 32). Aussi nous a-t-il été possible de faire ressortir le caractère social des attitudes, ce qu'une approche purement individualiste n’aurait sans doute pas été en mesure de faire. Il faut souligner qu'au moment où le recueil des données a été effectué, l'immigration était un objet particulièrement sensible de l'agenda politique, laissant supposer qu'à une autre période les mêmes outils auraient pu conduire à des résultats sensiblement différents. Aussi faut-il se concentrer sur les discours et canaux de communication pour comprendre la dimension sociale des attitudes et rendre compte de leur intégration dans les dynamiques sociales. Cette démarche peut se révéler fructueuse afin d’approfondir, notamment, le thème le plus intéressant du champ d'étude des attitudes: celui du changement (Montmollin, 2003 [1984], p. 90). Sans rien soustraire au postulat classique de la psychologie - selon lequel le changement d’attitude est un 
processus complexe car les prises de position de l'individu envers l'environnement s'enracinent dans sa vision la plus globale de la réalité - nous pouvons ajouter que ce changement serait d'autant plus difficile s'il n'était pas accompagné d'une mutation des RS attachées à l'objet en question. Comment en effet peut-on modifier les attitudes si la base socio-cognitive par laquelle elles prennent forme reste identique? Et comment peut-on changer d'opinion si les arguments discursifs disponibles ne sont jamais modifiés?

Sur ce point, il serait utile d’apporter une précision, de manière à échapper à l'écueil du déterminisme culturel auquel cette thèse pourrait conduire. Le discours public et les stratégies de communication, nonobstant leur dimension supra-individuelle, restent in fine des produits éminemment individuels, activés selon des logiques visant à satisfaire les intérêts des groupes. Les deux RS de l'immigré relevées dans notre étude résultent alors de discours propagés par les moyens de communication fournissant les «ingrédients » à la communication interindividuelle. C'est à travers cette dernière que les représentations se répandent entre les groupes, au moyen d'échanges d'opinions entre les individus. Farr va encore plus loin quand il affirme quaujourd'hui le caractère social des représentations dérive du fait qu’elles sont «véhiculées par les médias » (2003 [1984], p. 386). C’est pour cette raison que l'auteur fait prévaloir «tout l'intérêt de l'analyse du contenu des médias pour l'étude des représentations sociales » (ibid.). Les RS constituent alors une sorte de «vocabulaire de motifs » disponible, qui permet à l'acteur de s'orienter et se positionner face à l'environnement. Dans le cadre de cette étude, nous avons vu que la prise de distance par rapport aux groupes minoritaires s'opère par des arguments et des discours qui alimentent deux différents types de préjugés.

Enfin, nous avons souhaité soutenir l'idée que l'étude conjointe des RS et des attitudes est de nature à ancrer ces dernières dans la trame des rapports sociaux caractérisant un contexte donné. Afin de poursuivre dans cette voie, il serait cependant nécessaire d'ouvrir l’analyse des attitudes aux différentes techniques sociologiques et pyschosociales, car les échelles d’attitudes classiques ne permettent de saisir que la partie la plus superficielle des prises de positions individuelles envers l'environnement. Quant à l'identification des RS, elle 
permet d'approfondir l'aspect social des attitudes en les contextualisant, dans l'objectif de faire ressortir les discours dont elles sont le vecteur.

Aussi, pensons-nous que cette analyse ouvre des pistes intéressantes pour l'étude sociologique des relations entre les groupes et leur intégration au sein des dynamiques socio-politiques du pouvoir dans la société.

\section{BIBLIOGRAPHIE}

ABOUD E. Frances, DOYLE Anna-Beth., 1988, "Parental and Peer Influence on

Children's Racial Attitudes", International Journal of Intercultural Relations, vol.20, n॰3-4, p. 37I-383.

ABRIC Jean-Claude, 1987, Coopération, compétition et représentation sociale, Cousset, DelVal.

-, 2003, Méthodes d'études des représentations sociales, Paris, Érès.

ALLPORT G. Willard, 1935, "Attitudes", A Handbook of Social Psychology, Worchester, Clark University Press.

ALTMEYER Bob, 1998, "The other 'authoritarian personality"', in Marc Zanna, (dir.), Advances in Experimental and Social Psychology, San Diego, Academic Press.

AMBROSINI Maurizio, 2005, Sociologia dell'immigrazione, Bologna, II Mulino.

BAERVELD Chris, VAN DUIJN Marijtje, VERMEIJ Lotte, VAN HEMERT A. Dianne, 2004, "Ethnic boundaries and personal choice. Assessing the influence of individual inclinations to choose intra-ethnic relationships on pupils' networks", Social Networks, vol. 26, p. 55-74.

BLUM Alain, 1998, «Comment décrire les immigrés? À propos de quelques recherches sur l'immigration», Population, 53/3, p.569-588.

BLUMER Herbert, 1958, "Race Prejudice As a Sens of Group Position", Pacific Sociological Review, I/ I, p. 3-7.

BOLAFFI Guido, 200I, I confini del patto, Torino, Einaudi Editore.

BORGADUS S. Emory, 1931, "Mesuring social distance", Journal of Applied Sociology, $n^{\circ} 9,25$, p. 299-308. 
BRÉCHON Pierre, TCHERNIA Jean-François, 2009, La France à travers ses valeurs, Paris, Armand Colin.

CALAVITA Kitty, 2005, Immigrants at the margins: law, race, and exclusion in Southern Europe, Cambridge, Cambridge University Press.

CAPONIO Tiziana, 2006, Città italiane e immigrazione. Discorso pubblico e politiche a Milano, Bologna e Napoli, Bologna, II Mulino.

CARITAS/MIGRANTES, 2008, Dossier Statistico Immigrazione 2008, Roma, Anterem. -, 2010, Immigrazione. Dossier Statistico Immigrazione 2010, Roma, Idos.

COENDERS Marcel, LUBBERS Marcel, SCHEEPERS Peer, "Opposition to Civil Rights for Legal Migrants in Central and Eastern Europe", in East European Politics \& Societies, XXIII, n², 2005, p. I6I-184.

DAL LAGO Alessandro, 1999, «La tautologia della paura», Rassegna Italiana di Sociologia, vol. XXXX, n I, p. 5-4I.

-, 2004, Esistono davvero i conflitti tra culture? Una riflessione storico-metodologica, in Galli Carlo (dir.), Multiculturalismo. Ideologie e sfide, Bologna, II Mulino,

DECONCHY Jean Pierre, 2003 [1984], «Croyances et Idéologies», in Serge Moscovici (dir.), Psychologie sociale, Paris, PUF «Quadriges», p. 33I-355.

DEJAEGHERE Yves, HOOGHE Marc, CLAES Ellen, 20II, "Do ethnically diverse schools reduce ethnocentrism? A two-years panel study among majority group late adolescents in Belgian Schools", International Journal of Intercultural Relations, (à paraître).

DEMARIS Alfred, 1992, "Logit Modeling Practical Application", Sage University Paper, 86, Quantitative Applications in the Social Sciences, ENAP HA I3 QI.86; UQAH HB |4| D45; UQTR HB |4| D46.

DURKHEIM Émile, 1968 [1912], Les Formes élémentaires de la vie religieuse, Paris, PUF.

DURIEZ Bart, SOENENS Bart, 2009, "The intergenerational transmission of racism: The role of Right-Wing Authoritarianism and Social Dominance Orientation", Journal of Research in Personality, vol. 43, n 5, p. 906-909.

EAGLY H. Alice, CHAIKEN Shelly, 1993, The Psychology of Attitudes, Fort Worth Tex, Harcourt Brace Jovanavich. 
FARR M. Robert, 2003 [1984], «Les Représentations sociales»,

in Serge Moscovici (dir.), Psychologie sociale, Paris, PUF, «Quadriges », p. 379-389.

FLAMENT Claude, 1994, «Structure, dynamique et transformations des représentations sociales», in Jean-Claude Abric (dir.), Pratiques sociales et représentations, Paris, PUF, p. 37-57.

FREUD Sigmund, 1905, [2010], Trois essais sur la théorie sexuelle, Paris, Gallimard, «Folio».

GALLAND Olivier, 2007, Sociologie de la jeunesse, Paris, Armand Colin.

-, 2009, Les Jeunes, Paris, La Découverte, «Repères».

HEWSTONE Miles, STROBE Wolfgang, JONAS Klaus, VOCl Alberto, 2008, Introduction to social Psychology. A European Perspective, Oxford, Blackwell.

HOOGHE Marc, WILKENFELD Britt, 2008, "The stability of political attitudes and behaviors across adolescence and early adulthood: a comparison of survey data on adolescents and young adults in eight countries", Journal of Youth and Adolescence, 37/2, p. $155-167$.

INGLEARTH Ronald, 1997, Modernization and Postmodernization, Princeton, Princeton University Press.

INSTITUT NATIONAL DES ÉTUDES STATISTIQUES ET ÉCONOMIQUES, recensements de la population, en ligne: http://www.insee.fr/fr/themes/ tableau.asp?reg_id =0\&ref_id =NATTEF02I3|

INTERNATIONAL SOCIAL SURVEY PROGRAMM, National Identity, 2003, en ligne: http://www.za.uni-koeln.de/data/en/issp/questionnaires/q2003/CH2003_l.pdf.

JASPARS Jos, FRASER Colin, 1984, «Attitudes and social representations», in Robert M. Farr, Serge Moscovici (dir.), Social Representations, Cambridge, Cambridge University Press, p. 101-123.

JODELET Denise, 2003 [1984], «Représentation sociale: phénomènes, concept et théorie», in Serge Moscovici, Psychologie sociale, Paris, PUF, «Quadriges», p. 357-378.

LAPEYRONNIE Didier, 2008, Ghetto urbain. Ségrégation, violence, pauvreté en France aujourd'hui, Paris, Robert Laffont.

LE BRAS Hervé, 1998, Le Démon des origines, L'Aube, Paris. 
LE GOAZIOU Vincent, MUCCHIELLI Laurent, 2005, Quand les banlieues brûlent... Retour sur les émeutes de novembre 2005, Paris, La Découverte.

LEVY-BRUHL Herman, 1963 [1927], L'Âme primitive, Paris, PUF.

MANNONI Pierre, 2006, Les Représentations sociales, Paris, PUF, «Que sais-je?».

MC LAREN Lauren, 2003, "Anti-Immigrat Prejudice in Europe: Contact, Threat, Perception and Preferences for the Exclusion of Migrants", Social Forces, III, 2003, 81, p. 909-936.

MILLS-AFFIF Eduard, Filmer les immigrés. Les représentations audiovisuelles de l'immigration à la télévision française 1960-1984, Bruxelles, De Boeck.

MONTMOLLIN Germaine, 2003 [1984], «Le changement d'attitude», in Serge Moscovici (dir.), Psychologie sociale, Paris, PUF, «Quadriges», p. 91-138.

MORCELLINI Marco (dir.), 2009, Ricerca nazionale su immigrazione e asilo nei media italiani, Roma, Facoltà di Scienze della Formazione, Università la Sapienza.

MORICE Alain, 2005, Les émeutes urbaines d'octobre-novembre 2005 en France: comprendre avant de juger, Pajol: http://www.pajol.eu.org/article905.html [consulté le 28 février 20I0].

MOSCOVICI Serge, VIGNAUX George, 1994, «Le concept de thêmata», in Christian Guimelli (dir.), Structures et transformations des représentations sociales, Neuchâtel, Delachaux et Niestlé.

NOIRIEL Gerard, 2009, «"Color blindness" et construction des identités dans l'espace public français», in Didier Fassin, Eric Fassin (dir.), De la question sociale à la question raciale. Représenter la société française, Paris, La Découverte, «Poche», p. 166-182.

PIAGET Jean, 2005 [1947], La Représentation du monde chez l'enfant, Paris, PUF.

REINDERS Hans, 2003, «Politische sozialization in der adoleszenz. Eine RE-Interpretation quantitativer Längschnittunersuchunge in Deutschland», Zeitschrift Für Entwicklungspychologie und Pädagogische Psychologie, 35 /2, p.98-II0.

RATEAU Patrick, 2000, «Idéologie, représentation sociale et attitude: étude expérimentale de leur hiérarchie», Revue Internationale de Psychologie Sociale, n०13, vol. I, p. 29-57. 
ROSENBERG J. Morris, HOVLAND I. Carl, in Martin Fishbein, Icek Ajzen, 1975, Belief, Attitude, intention and behavior. An introduction to theory and research, Massachusetts, Addison-Wesley Publishing Company.

SAYAD Abdelmalek, 2006, L'Immigration ou les paradoxes de l'altérité, Paris, Raisons d'agir.

SCHNAPPER Dominique, 2008, «Les enjeux démocratiques de la statistique ethnique», Revue française de sociologie, n49-I, p. 133-139.

SCHOR Ralph, 1996, Histoire de l'immigration en France. De la fin du XIX siècle à nos jours, Paris, Armand Colin.

SECA Jean-Marie, 200I, Les Représentations sociales, Paris, Armand Colin.

SHERIF Muzafer, 1967a, Group conflict and cooperation, London, Routledge \& Kegan.

-, 1967b, Social interaction: Process and Products, Chicago, Aldine.

SNIDERMAN M. Paul, PERI Paolo, FIGUEIREDO J. P. Rui, PIAZZA J. Thomas, 2002, The Outsiders. Prejudice and Politics in Italy, Princeton-New Jersey, Princeton University Press.

STEPHAN G. Walter, STEPHAN W. Cookie, 1996, Intergroup relations, Boulder, Westview Press.

STREIFF-FENART Jocelyne, 2009, «Le "modèle républicain" et ses Autres: construction et évolution des catégories de l'altérité en France», Migrations Société, vol. XXI, n 122, p. 215-236.

THOMAS I. William, ZNANIECKI Florian, 1958, [1918-1920], The Polish Peasant in Europe and America, vol. I, New York, Dover Publications (vol. 2).

THOMAS Raymond, ALAPHILIPPE Daniel, 1993, Les Attitudes, Paris, PUF, «Que sais-je?».

THURSTONE L. Leon, 1928, "Attitudes can be measured", The American Journal of Sociology, IV, n॰33, p. 529-554.

TRIBALAT Michèle, 1995, Faire France. Une enquête sur les immigrés et leurs enfants, Paris, La Découverte. 
VERMEIJ Lotte, VAN DUIJN Marijtje, BAERVELDT Chris, 2009, "Ethnic segregation in context: Social discrimination among native Dutch pupils and their ethnic minority classmate", Social Networks, vol. 31, p. 230-239.

WELDON A. Steven, 2006, "The institutional context of tolerance for ethnic minorities: A comparative, multilevel analysis of Western Europe", American Journal of Political Science, 50/2, p.331-349.

ZINCONE Giovanna, 2005, «Cittadinanza e migrazioni: un'applicazione al caso italiano », in Massimo Livi Bacci (dir.), L'incidenza economica dell'immigrazione, Torino, Giappichelli, p. 374-403. 